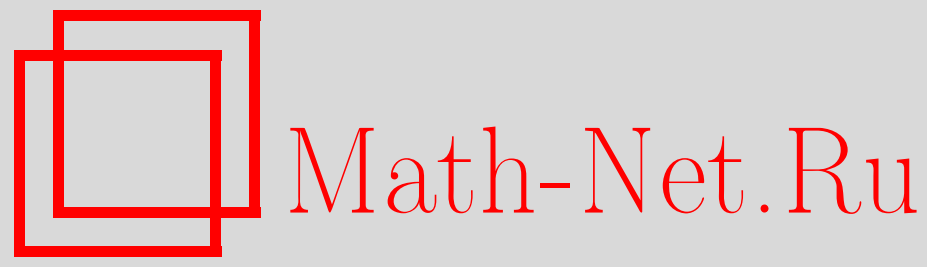

А. В. Казейкина, Отсутствие солитонов с достаточной алгебраической локализацией для уравнения Веселова-Новикова на ненулевом уровне энергии, Функи. анализ и его прил., 2014, том 48, выпуск $1,30-45$

DOI: https://doi.org/10.4213/faa3133

Использование Общероссийского математического портала MathNet.Ru подразумевает, что вы прочитали и согласны с пользовательским соглашением http://www.mathnet.ru/rus/agreement

Параметры загрузки:

IP : 34.239 .49 .27

26 апреля 2023 г., 12:10:07

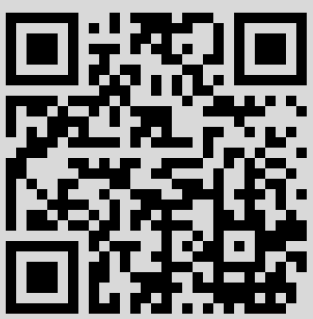


Функционалъный анализ и его приложения

2014, т. 48, вып. 1, с. 30-45

УДК 517.95

\title{
Отсутствие солитонов с достаточной алгебраической локализацией для уравнения Веселова-Новикова на ненулевом уровне энергии
}

\author{
(c) 2014. А. В. КАЗЕЙКИНА
}

В работе показано, что уравнение Веселова-Новикова (аналог уравнения КдФ в размерности $2+1$ ) на положительном и отрицательном уровнях энергии не имеет солитонов с пространственной локализацией выше, чем $O\left(|x|^{-3}\right)$, при $|x| \rightarrow \infty$.

\section{$\S 1$. Введение}

В данной работе рассматривается уравнение Веселова-Новикова

$$
\begin{gathered}
\partial_{t} v=4 \operatorname{Re}\left(4 \partial_{z}^{3} v+\partial_{z}(v w)-E \partial_{z} w\right), \\
\partial_{\bar{z}} w=-3 \partial_{z} v, \quad v=\bar{v}, \quad E \in \mathbb{R}, \\
v=v(x, t), \quad w=w(x, t), \quad x=\left(x_{1}, x_{2}\right) \in \mathbb{R}^{2}, t \in \mathbb{R},
\end{gathered}
$$

где

$$
\partial_{t}=\frac{\partial}{\partial t}, \quad \partial_{z}=\frac{1}{2}\left(\frac{\partial}{\partial x_{1}}-i \frac{\partial}{\partial x_{2}}\right), \quad \partial_{\bar{z}}=\frac{1}{2}\left(\frac{\partial}{\partial x_{1}}+i \frac{\partial}{\partial x_{2}}\right) .
$$

Уравнение (1.1) является математически наиболее естественным $(2+1)$-мерным аналогом классического уравнения Кортевега-де Фриза. Если $v=v\left(x_{1}, t\right)$, $w=w\left(x_{1}, t\right)$, то уравнение (1.1) сводится к уравнению КдФ. Кроме того, уравнение (1.1) интегрируемо при помощи преобразования рассеяния для двумерного уравнения Шрёдингера

$$
\begin{gathered}
L \psi=E \psi, \quad E=E_{\text {fixed }}, \\
L=-\Delta+v(x, t), \quad \Delta=4 \partial_{z} \partial_{\bar{z}}, \quad x \in \mathbb{R}^{2} .
\end{gathered}
$$

Отметим также, что при $E \rightarrow \pm \infty$ уравнение (1.1) переходит в другой известный $(2+1)$-мерный аналог уравнения КдФ, уравнение Кадомцева-Петвиашвили (КП-I и КП-ІІ соответственно; см., например, [12] и приведенные там ссылки).

Уравнение (1.1) содержится неявно в [22] как уравнение, имеющее следующее представление:

$$
\frac{\partial(L-E)}{\partial t}=[L-E, A]+B(L-E),
$$

где $L$ - оператор соответствующей задачи рассеяния, $A, B$ - некоторые подходящие дифференциальные операторы, $[\cdot, \cdot \cdot]$ обозначает коммутатор. Для частного случая двумерного оператора Шрёдингера из (1.3) операторы $A$ и $B$ в явной форме

$$
A=-8 \partial_{z}^{3}-2 w \partial_{z}-8 \partial_{\bar{z}}^{3}-2 \bar{w} \partial_{\bar{z}}, \quad B=2 \partial_{\bar{z}} w+2 \partial_{\bar{z}} \bar{w},
$$


где $w$ определяется в $(1.1 \mathrm{~b})$, и соответствующее эволюционное уравнение $(1.1)$ были получены в [25], [26], где уравнение (1.1) далее изучалось в периодической постановке.

Солитоны и асимптотическое при больших временах поведение решений уравнения Веселова-Новикова с достаточной пространственной локализацией изучались в серии работ [13], [11], [24], [16], [19]-[21]. В [19], [16] было показано, что в регулярном случае, т. е. когда данные рассеяния невырожденны при фиксированной ненулевой энергии (и в безотражательном случае на положительном уровне энергии), соответствующие решения уравнения Веселова-Новикова не содержат локализованных солитонов в асимптотике при больших временах. В общем случае в [24], [21] было показано, что уравнение Веселова-Новикова на ненулевом уровне энергии не допускает экспоненциально локализованных солитонов. Семейство алгебраически локализованных солитонов для уравнения Веселова-Новикова на положительном уровне энергии было построено в [11] (см. также обсуждение в [20]). Эти солитоны являются рациональными функциями, убывающими, как $O\left(|x|^{-2}\right)$, при $|x| \rightarrow \infty$.

Отметим, что уравнение КП-І имеет солитонные решения, и эти решения убывают, как $O\left(|x|^{-2}\right)$, при $|x| \rightarrow \infty$. Уравнение KП-II, напротив, не обладает локализованными солитонными решениями. По вопросу существования и отсутствия локализованных солитонных решений уравнений KП-I, KП-II и их обобщений см. [4]; симметрии и скорости убывания этих решений были изучены в [5]. Дополнительные результаты об интегрируемых $(2+1)$-мерных системах, допускающих локализованные солитонные решения, можно найти в [1], [3], [8], [9], см. также приводимые там ссылки.

В этой работе мы рассматриваем регулярные, достаточно локализованные решения уравнения (1.1), удовлетворяющие следующим условиям:

- $v, w \in C\left(\mathbb{R}^{2} \times \mathbb{R}\right), v(\cdot, t) \in C^{4}\left(\mathbb{R}^{2}\right)$ для любого $t \in \mathbb{R}$;

- $\left|\partial_{x}^{j} v(x, t)\right| \leqslant \frac{q(t)}{(1+|x|)^{3+j+\varepsilon}}, j=\left(j_{1}, j_{2}\right) \in(\mathbb{N} \cup 0)^{2}, j_{1}+j_{2} \leqslant 4$, при некоторых $q(t)>0, \varepsilon>0$;

- $|w(x, t)| \rightarrow 0$ при $|x| \rightarrow \infty, t \in \mathbb{R}$.

Будем говорить, что решение уравнения (1.1) является солитоном, если $v(x, t)=$ $V(x-c t)$ для некоторого $c=\left(c_{1}, c_{2}\right) \in \mathbb{R}^{2}$. Основной результат данной статьи состоит в следующем.

Теорема 1.1. Пусть $(v, w)$ - солитонное решение уравнения (1.1) при $E \neq 0$, удовлетворяющее условиям (1.6)-(1.8). Тогда $v \equiv 0, w \equiv 0$.

Доказательство этой теоремы основано, в частности, на рассмотрении специальных собственных функций двумерного оператора Шрёдингера, введенных в [6], [2], и на идеях, предложенных в [24].

Заметим, что теорема 1.1 для случая нулевой энергии была доказана в [17] для потенциалов кондуктивного типа.

В 22 приводятся, в частности, некоторые известные сведения из теории прямой и обратной задачи рассеяния для двумерного уравнения Шрёдингера на ненулевом уровне энергии (см. [23], [12] и приведенные там ссылки). Кроме того, мы вводим для ненулевого уровня энергии аналоги некоторых данных рассея- 
ния, рассмотренных в [2]. Основной результат (теорема 1.1) доказывается в 33. В $\S 4$ содержатся доказательства некоторых предварительных лемм.

Данная работа была выполнена в рамках научного исследования под руководством Р. Г. Новикова.

\section{§2. Данные рассеяния и уравнения обратной задачи рассеяния}

Рассмотрим уравнение Шрёдингера на плоскости

$$
\begin{gathered}
L \psi=E \psi, \quad E=E_{\text {fixed }} \in \mathbb{R} \backslash 0, \\
L=-\Delta+v, \quad \Delta=4 \partial_{z} \partial_{\bar{z}}, \quad v=v(x), \quad x \in \mathbb{R}^{2},
\end{gathered}
$$

с потенциалом $v$, удовлетворяющим следующим условиям:

$$
\begin{aligned}
& v(x)=\overline{v(x)}, \quad v(x) \in L^{\infty}\left(\mathbb{R}^{2}\right), \\
& \left|\partial_{x_{1}}^{j_{1}} \partial_{x_{2}}^{j_{2}} v(x)\right|<q(1+|x|)^{-3-\varepsilon} \\
& \quad \text { для некоторых } q>0, \varepsilon>0, \text { где } j_{1}, j_{2} \in \mathbb{N} \cup 0, j_{1}+j_{2} \leqslant 3 .
\end{aligned}
$$

Для уравнения (2.1) при $E>0$ рассмотрим его собственные функции $\psi^{+}(x, k)$, определенные при $k \in \mathbb{R}^{2}, k^{2}=E$, и удовлетворяющие асимптотике

$$
\psi^{+}(x, k)=e^{i k x}-i \pi \sqrt{2 \pi} e^{-i \pi / 4} f\left(k,|k| \frac{x}{|x|}\right) \frac{e^{i|k||x|}}{\sqrt{|k||x|}}+o\left(\frac{1}{\sqrt{|x|}}\right), \quad|x| \rightarrow \infty,
$$

для некоторой априорно неизвестной функции $f$. Функция $f$ называется $а$ мплитудой рассеяния потенциала $v$. Если $f(k, l) \equiv 0$ при $k, l \in \mathbb{R}^{2}, k^{2}=l^{2}=E$, то соответствующий потенциал называется прозрачным (или безотражательным) при фиксированной энергии $E>0$. В данной работе рассматриваются исключительно прозрачные потенциалы, поскольку, как было показано в [24], солитоны уравнения Веселова-Новикова на положительном уровне энергии являются прозрачными потенциалами (см. также лемму 3.4).

Для уравнения (2.1) при $E \in \mathbb{R} \backslash 0$ рассмотрим также собственные функции Фаддеева $\psi(x, k)$, определенные при $k \in \Sigma_{E}$, где

$$
\Sigma_{E}= \begin{cases}\left\{k \in \mathbb{C}^{2}: k^{2}=E, \operatorname{Im} k \neq 0\right\}, & \text { если } E>0, \\ \left\{k \in \mathbb{C}^{2}: k^{2}=E\right\}, & \text { если } E<0,\end{cases}
$$

и удовлетворяющие асимптотике

$$
\psi(x, k)=e^{i k x}(1+o(1)), \quad|x| \rightarrow \infty
$$

(см. [6], [23], [12]).

Наконец, для уравнения (2.1) при $E \in \mathbb{R} \backslash 0$ мы также рассмотрим собственные функции $\varphi(x, k)$, определенные при $k \in \Sigma_{E}$ и удовлетворяющие асимптотике

$$
\varphi(x, k)=e^{i k x}\left(k_{1} x_{2}-k_{2} x_{1}+o(1)\right), \quad|x| \rightarrow \infty .
$$

Эти функции являются аналогами решений, введенных в [2] для случая нулевой энергии. 
Далее будет удобно без потери общности предположить, что $E= \pm 1$ (общий случай сводится к данному преобразованием масштабирования), и ввести новые переменные

$$
z=x_{1}+i x_{2}, \quad \lambda=\frac{k_{1}+i k_{2}}{\sqrt{E}} .
$$

Отметим, что $k_{1}=\frac{\sqrt{E}}{2}\left(\lambda+\frac{1}{\lambda}\right), k_{2}=\frac{i \sqrt{E}}{2}\left(\frac{1}{\lambda}-\lambda\right)$.

$\mathrm{B}$ новых переменных $z \in \mathbb{C}, \lambda \in \mathbb{C} \backslash 0$ функции $\psi$ и $\varphi$ определяются как решения уравнения (2.1) со следующим асимптотическим поведением:

$$
\begin{array}{ll}
\psi(z, \lambda)=e^{\frac{i \sqrt{E}}{2}(\lambda \bar{z}+z / \lambda)} \mu(z, \lambda), & \mu(z, \lambda)=1+o(1) \quad \text { при }|z| \rightarrow \infty, \\
\varphi(z, \lambda)=e^{\frac{i \sqrt{E}}{2}(\lambda \bar{z}+z / \lambda)} \nu(z, \lambda), & \nu(z, \lambda)=\frac{i \sqrt{E}}{2}\left(\lambda \bar{z}-\frac{1}{\lambda} z\right)+o(1) \quad \text { при }|z| \rightarrow \infty .
\end{array}
$$

Функции $\mu(z, \lambda)$ и $\nu(z, \lambda)$, возникающие в приведенных выше формулах, также могут быть определены как решения интегральных уравнений

$$
\begin{aligned}
& \mu(z, \lambda)=1+\iint_{\mathbb{C}} g(z-\zeta, \lambda) v(\zeta) \mu(\zeta, \lambda) d \operatorname{Re} \zeta d \operatorname{Im} \zeta \\
& \nu(z, \lambda)=\frac{i \sqrt{E}}{2}\left(\lambda \bar{z}-\frac{1}{\lambda} z\right)+\iint_{\mathbb{C}} g(z-\zeta, \lambda) v(\zeta) \nu(\zeta, \lambda) d \operatorname{Re} \zeta d \operatorname{Im} \zeta,
\end{aligned}
$$

где

$$
g(z, \lambda)=-\left(\frac{1}{2 \pi}\right)^{2} \iint_{\mathbb{C}} \frac{e^{\frac{i}{2}(p \bar{z}+\bar{p} z)}}{p \bar{p}+\sqrt{E}(\lambda \bar{p}+p / \lambda)} d \operatorname{Re} p d \operatorname{Im} p
$$

и $z \in \mathbb{C}, \lambda \in \mathbb{C} \backslash 0$, а также, если $E>0$, то $|\lambda| \neq 1$.

В терминах $m(z, \lambda)=(1+|z|)^{-(2+\varepsilon / 2)} \mu(z, \lambda)$ и $n(z, \lambda)=(1+|z|)^{-(2+\varepsilon / 2)} \nu(z, k)$ уравнения $(2.8)$ и $(2.9)$ соответственно записываются в следующем виде:

$$
\begin{aligned}
& m(z, \lambda)=(1+|z|)^{-(2+\varepsilon / 2)} \\
& \quad+\iint_{\mathbb{C}}(1+|z|)^{-(2+\varepsilon / 2)} g(z-\zeta, \lambda) \frac{v(\zeta)}{(1+|\zeta|)^{-(2+\varepsilon / 2)}} m(\zeta, \lambda) d \operatorname{Re} \zeta d \operatorname{Im} \zeta, \\
& n(z, \lambda)=\frac{i \sqrt{E}}{2}\left(\lambda \bar{z}-\frac{1}{\lambda} z\right)(1+|z|)^{-(2+\varepsilon / 2)} \\
& \quad+\iint_{\mathbb{C}}(1+|z|)^{-(2+\varepsilon / 2)} g(z-\zeta, \lambda) \frac{v(\zeta)}{(1+|\zeta|)^{-(2+\varepsilon / 2)}} n(\zeta, \lambda) d \operatorname{Re} \zeta d \operatorname{Im} \zeta .
\end{aligned}
$$

Интегральный оператор $A(\lambda)$ интегральных уравнений $(2.11),(2.12)$ является оператором Гильберта-Шмидта, а именно, $A(\cdot, \cdot, \lambda) \in L^{2}(\mathbb{C} \times \mathbb{C})$, где $A(z, \zeta, \lambda)$ - ядро Шварца интегрального оператора $A(\lambda)$, и $\left|\operatorname{Tr} A^{2}(\lambda)\right|<\infty$. Таким образом, модифицированный определитель Фредгольма $\Delta(\lambda)$ для $(2.11)$ и $(2.12)$ может быть определен следующим образом:

$$
\ln \Delta(\lambda)=\operatorname{Tr}(\ln (I-A(\lambda))+A(\lambda)) .
$$

Точный смысл данного определения приведен в [10]. Впервые определитель $\Delta$

2 Функциональный анализ и его приложения, т. 48, вып. 1 
для уравнений типа (2.11), (2.12) был рассмотрен в [7].

Также положим

$$
\mathscr{E}=\{\lambda \in \Sigma: \Delta(\lambda)=0\}
$$

где

$\Sigma=\mathbb{C} \backslash(0 \cup T)$, если $E>0, \quad$ и $\Sigma=\mathbb{C} \backslash 0$, если $E<0, \quad T=\{\lambda \in \mathbb{C}:|\lambda|=1\}$.

Множество $\mathscr{E}$ представляет собой множество значений $\lambda$, для которых не имеет места либо существование, либо единственность решения уравнения (2.1) с асимптотикой (2.6) (или, эквивалентно, решения уравнения (2.1) с асимптотикой (2.7)).

При $\lambda \in \mathbb{C} \backslash(\mathscr{E} \cup 0)$ определим следующие «данные рассеяния» для потенциала $v$ :

$$
\begin{aligned}
& a(\lambda)=\iint_{\mathbb{C}} v(\zeta) \mu(\zeta, \lambda) d \operatorname{Re} \zeta d \operatorname{Im} \zeta \\
& b(\lambda)=\iint_{\mathbb{C}} \exp \left(\frac{i \sqrt{E}}{2}\left(1+(\operatorname{sgn} E) \frac{1}{\lambda \bar{\lambda}}\right)((\operatorname{sgn} E) \zeta \bar{\lambda}+\lambda \bar{\zeta})\right) v(\zeta) \mu(\zeta, \lambda) d \operatorname{Re} \zeta d \operatorname{Im} \zeta,
\end{aligned}
$$

$\alpha(\lambda)=\iint_{\mathbb{C}} v(\zeta) \nu(\zeta, \lambda) d \operatorname{Re} \zeta d \operatorname{Im} \zeta$,

$\beta(\lambda)=\iint_{\mathbb{C}} \exp \left(\frac{i \sqrt{E}}{2}\left(1+(\operatorname{sgn} E) \frac{1}{\lambda \bar{\lambda}}\right)((\operatorname{sgn} E) \zeta \bar{\lambda}+\lambda \bar{\zeta})\right) v(\zeta) \nu(\zeta, \lambda) d \operatorname{Re} \zeta d \operatorname{Im} \zeta$.

Функции $a, b$ являются обобщенными данными рассеяния Фаддеева для двумерного уравнения Шрёдингера. Они также возникают при рассмотрении членов более высокого порядка в разложении (2.4). «Данные рассеяния» $\alpha, \beta$ для уравнения Шрёдингера на нулевом уровне энергии были введены в [2].

Теперь сформулируем некоторые свойства определенных выше функций, которые будут играть существенную роль при доказательстве основного результата.

Утверждение 2.1 (см. [15], [23], [21]). Пусть v удовлетворяет условиям (2.2). Тогда функиия $\Delta(\lambda)$ обладает следующими свойствами:

(1) $\Delta \in C\left(\bar{D}_{+}\right), \Delta \in C\left(\bar{D}_{-}\right)$, где $\bar{D}_{+}=D_{+} \cup \partial D_{+}, D_{+}=\{\lambda \in \mathbb{C}:|\lambda|<1\}$, $\bar{D}_{-}=D_{-} \cup \partial D_{-}, D_{-}=\{\lambda \in \mathbb{C}:|\lambda|>1\}$;

(2) $\Delta(\lambda) \rightarrow 1$ npu $|\lambda| \rightarrow \infty,|\lambda| \rightarrow 0$

(3) $\Delta$ вещественнозначна;

(4) $\Delta(\lambda)$ удовлетворяет $\bar{\partial}$-уравнению

$$
\frac{\partial \Delta}{\partial \bar{\lambda}}=-\frac{\operatorname{sgn}(\lambda \bar{\lambda}-1)}{4 \pi \bar{\lambda}}\left(a\left(-(\operatorname{sgn} E) \frac{1}{\bar{\lambda}}\right)-\hat{v}(0)\right) \Delta,
$$

где $\hat{v}(0)=\iint_{\mathbb{C}} v(\zeta) d \operatorname{Re} \zeta d \operatorname{Im} \zeta, \lambda \in \mathbb{C} \backslash(T \cup \mathscr{E} \cup 0), T=\{\lambda \in \mathbb{C}:|\lambda|=1\} ;$

(5) $\Delta(\lambda)=\Delta\left(-(\operatorname{sgn} E) \frac{1}{\lambda}\right), \lambda \in \mathbb{C} \backslash 0$;

(6) если $E<0$ или если $E>0$ и $v$ является прозрачным потенииалом, то $\Delta \equiv$ const на $T=\{\lambda \in \mathbb{C}:|\lambda|=1\}$. 
Отметим, что в общем случае $\Delta \notin C(\mathbb{C})$ при $E>0$. В этом случае $\Delta$ на $\bar{D}_{ \pm}$ рассматривается как непрерывное продолжение с $D_{ \pm}$.

Если $v$ удовлетворяет предположениям (2.2), то функции $a(\lambda), b(\lambda), \alpha(\lambda)$, $\beta(\lambda)$ являются непрерывными на $\mathbb{C} \backslash(\mathscr{E} \cup 0)$. Отметим также (см. [15]), что

$$
a(\lambda) \rightarrow \hat{v}(0) \quad \text { при } \lambda \rightarrow 0, \lambda \rightarrow \infty .
$$

Если данные рассеяния $а$ определены на окружности $T$, то их поведение на ней описывается следующим образом:

$a \equiv 0$ на $T$, если $E>0$ и $v$ является прозрачным потенциалом (см. [12]),

$a \equiv b$ на $T$, если $E<0$.

Если $v$ удовлетворяет предположениям (2.2) и при положительной энергии $v$ является прозрачным потенциалом, т. е. $f \equiv 0$ при фиксированной энергии, то функция $\mu(z, \lambda)$, определенная в $(2.8)$, обладает следующими свойствами (см. [14], [24], [12] и приводимые там ссылки):

$$
\begin{gathered}
\mu(z, \lambda) \text { является непрерывной по } \lambda \text { на } \mathbb{C} \backslash(0 \cup \mathscr{E}) ; \\
\frac{\partial \mu(z, \lambda)}{\partial \bar{\lambda}}=r(z, \lambda) \overline{\mu(z, \lambda)}, \\
r(z, \lambda)=r(\lambda) \exp \left(-\frac{i \sqrt{E}}{2}\left(1+(\operatorname{sgn} E) \frac{1}{\lambda \bar{\lambda}}\right)((\operatorname{sgn} E) \bar{\lambda} z+\lambda \bar{z})\right), \\
r(\lambda)=\frac{\operatorname{sgn}(\lambda \bar{\lambda}-1)}{4 \pi \bar{\lambda}} b(\lambda)
\end{gathered}
$$

при $\lambda \in \mathbb{C} \backslash(0 \cup \mathscr{E} \cup T)$, где $T=\{\lambda \in \mathbb{C}:|\lambda|=1\}$;

$$
\mu \rightarrow 1 \text { при } \lambda \rightarrow \infty, \lambda \rightarrow 0 .
$$

Уравнения обратной задачи рассеяния (2.22) вместе с условиями (2.21) и (2.23) однозначно определяют функцию $\mu$ по невырожденным данным рассеяния $b$, т. е. в случае, когда $\mathscr{E}=\varnothing$. Потенциал $v$ (прозрачный в случае положительной энергии) может быть определен по формуле

$$
v(z)=2 i \sqrt{E} \frac{\partial \mu_{-1}(z)}{\partial z},
$$

где $\mu_{-1}(z)$ определяется через следующее асимптотическое разложение:

$$
\mu(z, \lambda)=1+\frac{\mu_{-1}(z)}{\lambda}+o\left(\frac{1}{|\lambda|}\right) \quad \text { при } \lambda \rightarrow \infty .
$$

\section{§3. Доказательство теоремы 1.1}

Сформулируем сначала несколько предварительных лемм. Доказательства лемм $3.2,3.3$ приводятся в $\S 4$.

Лемма 3.1. Пусть $v \in C^{4}(\mathbb{C}) u$

$$
\left|\partial_{z}^{j_{1}} \partial_{\bar{z}}^{j_{2}} v(z)\right|<\frac{q}{(1+|z|)^{j+3+\varepsilon}}, \quad j_{1}, j_{2} \in\{0 \cup \mathbb{N}\}, j=j_{1}+j_{2} \leqslant 4
$$


для любых $z \in \mathbb{C}$ и некоторых $q>0, \varepsilon>0$. Пусть $w$ определяется следующим образом:

$$
\partial_{\bar{z}} w=-3 \partial_{z} v, \quad w(z) \rightarrow 0 \quad n p u \quad z \rightarrow \infty .
$$

Тогда

$$
\begin{aligned}
w(z) & =\frac{3 \hat{v}(0)}{\pi z^{2}}+O\left(\frac{1}{|z|^{3}}\right) \quad \text { nри } z \rightarrow \infty, \\
\partial_{z} w(z) & =-\frac{6 \hat{v}(0)}{\pi z^{3}}+O\left(\frac{1}{|z|^{4}}\right) \quad \text { nрu } z \rightarrow \infty,
\end{aligned}
$$

где $\hat{v}(0)=\iint_{\mathbb{C}} v(\zeta) d \operatorname{Re} \zeta d \operatorname{Im} \zeta$.

Доказательства представлений (3.2), (3.3) легко выводятся из формулы Тейлора для $W(\xi)=w(1 / \xi)$ и $-\xi^{2} W(\xi)=\left.\partial_{z} w(z)\right|_{z=1 / \xi}$ соответственно в окрестности $\xi=0$.

Обозначим через

$$
S(\lambda)=\{a(\lambda), b(\lambda), \alpha(\lambda), \beta(\lambda)\}, \quad \lambda \in \mathbb{C} \backslash(\mathscr{E} \cup 0),
$$

данные рассеяния для потенциала $v$, определенные в (2.14)-(2.17) в рамках уравнения (2.1).

Лемма 3.2. Пусть $v(z)$ - потенциал, удовлетворяющий условию (2.2), $\Delta(\lambda)$ - соответствующий ему модифицированный определитель Фредгольма, определяемый по формуле (2.13), $S(\lambda)$ - данные рассеяния для потенииала $v$, определенные при $\lambda \in \mathbb{C} \backslash(\mathscr{E} \cup 0)$. Тогда модифицированный определитель Фредгольма $\Delta_{\eta}(\lambda)$ и данные рассеяния $S_{\eta}(\lambda)$ для потеницала $v_{\eta}(z)=v(z-\eta)$ обладают следующими свойствами:

(1) $\Delta_{\eta}(\lambda)=\Delta(\lambda)$

(2) данные рассеяния $S_{\eta}(\lambda)$ определены при $\lambda \in \mathbb{C} \backslash(\mathscr{E} \cup 0)$ и связаны с $S(\lambda)$ формулами

$$
\begin{aligned}
& a_{\eta}(\lambda)=a(\lambda) \\
& b_{\eta}(\lambda)=\exp \left\{\frac{i \sqrt{E}}{2}\left(1+(\operatorname{sgn} E) \frac{1}{\lambda \bar{\lambda}}\right)((\operatorname{sgn} E) \bar{\lambda} \eta+\lambda \bar{\eta})\right\} b(\lambda), \\
& \alpha_{\eta}(\lambda)=\alpha(\lambda)+\frac{i \sqrt{E}}{2}\left(\lambda \bar{\eta}-\frac{1}{\lambda} \eta\right) a(\lambda), \\
& \beta_{\eta}(\lambda)=\exp \left\{\frac{i \sqrt{E}}{2}\left(1+(\operatorname{sgn} E) \frac{1}{\lambda \bar{\lambda}}\right)((\operatorname{sgn} E) \bar{\lambda} \eta+\lambda \bar{\eta})\right\} \\
& \times\left(\beta(\lambda)+\frac{i \sqrt{E}}{2}\left(\lambda \bar{\eta}-\frac{1}{\lambda} \eta\right) b(\lambda)\right) .
\end{aligned}
$$

Лемма 3.3. Пусть $(v, w)$ удовлетворяет уравнению (1.1) и условиям (1.6)(1.8). Пусть $S(\lambda, t)$ - данные рассеяния для $v$, определенные с помощью (3.4) для некоторого $\lambda \in \mathbb{C} \backslash(\mathscr{E} \cup 0)$ и всех $t \in \mathbb{R}$. Тогда эволючия этих данных 
рассеяния описывается следующим образом:

$$
\begin{aligned}
& a(\lambda, t)=a(\lambda, 0), \\
& b(\lambda, t)=\exp \left\{i(\sqrt{E})^{3}\left(\lambda^{3}+\frac{1}{\lambda^{3}}+(\operatorname{sgn} E)\left(\bar{\lambda}^{3}+\frac{1}{\bar{\lambda}^{3}}\right)\right) t\right\} b(\lambda, 0), \\
& \alpha(\lambda, t)=\alpha(\lambda, 0)+3 i(\sqrt{E})^{3}\left(\lambda^{3}-\frac{1}{\lambda^{3}}\right)(a(\lambda, 0)-\hat{v}(0)) t, \\
& \beta(\lambda, t)=\exp \left\{i(\sqrt{E})^{3}\left(\lambda^{3}+\frac{1}{\lambda^{3}}+(\operatorname{sgn} E)\left(\bar{\lambda}^{3}+\frac{1}{\bar{\lambda}^{3}}\right)\right) t\right\} \\
& \times\left(\beta(\lambda, 0)+3 i(\sqrt{E})^{3}\left(\lambda^{3}-\frac{1}{\lambda^{3}}\right) b(\lambda, 0) t\right) .
\end{aligned}
$$

Лемма 3.4 (см. [24]). Пусть (v,w) удовлетворяет уравнению (1.1) при некотором $E>0$ и условиям (1.6)-(1.8). Кроме того, пусть $v$ - солитон, т.е. $v(x, t)=V(x-c t)$ при некотором $c=\left(c_{1}, c_{2}\right) \in \mathbb{R}^{2}$. Тогда $f(k, l) \equiv 0, k, l \in \mathbb{R}^{2}$, $k^{2}=l^{2}=E>0$, где $f$ - амплитуда рассеяния потенииала $v$ в рамках уравнения Шрёдингера (1.3).

Заключительная часть доказательства теоремы 1.1 состоит в следующем. Прежде всего, если $(v, w)$ является солитонным решением уравнения (1.1) при некотором $E>0$, то $v$ является прозрачным потенциалом вследствие леммы 3.4 .

Далее, поскольку $(v, w)$ - солитон, то из леммы 3.2 следует, что множество $\mathscr{E}$ значений $\lambda \in \mathbb{C}$, для которых данные рассеяния $a(\lambda), b(\lambda), \alpha(\lambda), \beta(\lambda)$ не определены, не зависит от $t$.

Поскольку $(v, w)$ - солитон, динамика по времени его данных рассеяния $b$ описывается формулой

$$
b(\lambda, t)=\exp \left\{\frac{i \sqrt{E}}{2}\left(\left(\lambda+(\operatorname{sgn} E) \frac{1}{\bar{\lambda}}\right) \bar{c} t+\left((\operatorname{sgn} E) \bar{\lambda}+\frac{1}{\lambda}\right) c t\right)\right\} b(\lambda, 0),
$$

где используется обозначение $c=c_{1}+i c_{2}$ (см. формулу (3.6) леммы 3.2). Объединяя это выражение с формулой (3.10) из леммы 3.3, получаем

$$
\begin{aligned}
\exp \left\{i(\sqrt{E})^{3}\left(\lambda^{3}+\frac{1}{\lambda^{3}}+(\operatorname{sgn} E)\left(\bar{\lambda}^{3}+\frac{1}{\bar{\lambda}^{3}}\right)\right) t\right\} b(\lambda, 0) \\
\quad=\exp \left\{\frac{i \sqrt{E}}{2}\left(\left(\lambda+(\operatorname{sgn} E) \frac{1}{\bar{\lambda}}\right) \bar{c} t+\left((\operatorname{sgn} E) \bar{\lambda}+\frac{1}{\lambda}\right) c t\right)\right\} b(\lambda, 0) .
\end{aligned}
$$

Поскольку функции $\lambda, \bar{\lambda}, \lambda^{3}, \bar{\lambda}^{3}, 1 / \lambda, 1 / \bar{\lambda}, 1 / \lambda^{3}, 1 / \bar{\lambda}^{3}, 1$ являются линейно независимыми в любой открытой непустой окрестности любой точки из $\mathbb{C} \backslash 0$ и $b(\lambda, 0)$ непрерывна на $\mathbb{C} \backslash(\mathscr{E} \cup 0)$, получаем, что $b(\lambda, 0) \equiv 0$ на $\mathbb{C} \backslash(\mathscr{E} \cup 0)$.

Аналогично из (3.7), (3.11) получаем, что

$$
\alpha(\lambda, 0)+\frac{i \sqrt{E}}{2}\left(\lambda \bar{c}-\frac{1}{\lambda} c\right) t a(\lambda, 0)=\alpha(\lambda, 0)+3 i(\sqrt{E})^{3}\left(\lambda^{3}-\frac{1}{\lambda^{3}}\right) t(a(\lambda, 0)-\hat{v}(0)),
$$

или

$$
a(\lambda, 0)=\frac{3 i(\sqrt{E})^{3}\left(\lambda^{3}-\frac{1}{\lambda^{3}}\right) \hat{v}(0)}{3 i(\sqrt{E})^{3}\left(\lambda^{3}-\frac{1}{\lambda^{3}}\right)-\frac{i \sqrt{E}}{2}\left(\lambda \bar{c}-\frac{1}{\lambda} c\right)} .
$$


Докажем, что из представления (3.13) следует равенство $\hat{v}(0)=0$ и, таким образом, $a(\lambda, 0) \equiv 0$ при $\lambda \in \mathbb{C} \backslash(\mathscr{E} \cup 0)$.

Выражение (3.13) определено всюду за исключением точек, являющихся корнями уравнения

$$
3 i(\sqrt{E})^{3}\left(\lambda^{3}-\frac{1}{\lambda^{3}}\right)-\frac{i \sqrt{E}}{2}\left(\lambda \bar{c}-\frac{1}{\lambda} c\right)=0 .
$$

Очевидно, что уравнение (3.14) имеет шесть корней $\lambda_{j}, j=1, \ldots, 6$, с учетом кратности. Оно было подробно изучено в [19] (см. лемму 3.1 из [19]). В частности, было показано, что для любого значения параметра $c$

уравнение (3.14) имеет по меньшей мере два корня на $T=\{\lambda \in \mathbb{C}:|\lambda|=1\}$.

Рассмотрим два случая в зависимости от того, обращается ли $\Delta$ на $T$ в нуль или нет. Отметим, что, согласно п. (6) утверждения 2.1, если определитель $\Delta$ обращается в нуль в одной точке из $T$, то он тождественно равен нулю на $T$.

(i) $\Delta \neq 0$ на $T$.

В этом случае $a(\lambda, 0)$ и $b(\lambda, 0)$ определены на $T$. Значит, $b(\lambda, 0) \equiv 0$ на $T$. Из $(2.20)$ получаем, что $a(\lambda, 0) \equiv 0$ на $T$. В силу представления (3.13) и свойства (3.15) это может иметь место, только если $\hat{v}(0)=0$.

(ii) $\Delta \equiv 0$ на $T$.

Вспомним, что в силу п. (3) утверждения $2.1 \Delta \in \mathbb{R}$, а в силу п. (2) утверждения $2.1 \Delta(0)>0$. Рассмотрим

$$
l_{\varphi}=\left\{r e^{i \varphi}, 0 \leqslant r<r^{\prime} \leqslant 1: \Delta\left(r e^{i \varphi}\right)>0, \Delta\left(r^{\prime} e^{i \varphi}\right)=0\right\},
$$

где $\varphi \in(-\pi, \pi]-$ некоторый угол, такой, что $\varphi \neq \operatorname{Arg} \lambda_{j}, j=1, \ldots, 6$, где $\lambda_{j}-$ корни уравнения (3.14). Обозначим также через $\bar{l}_{\varphi}$ замыкание множества $l_{\varphi}$.

Положим

$$
u(\bar{\lambda})=-\frac{\operatorname{sgn}(\lambda \bar{\lambda}-1)}{4 \pi \bar{\lambda}}\left(a\left(-(\operatorname{sgn} E) \frac{1}{\bar{\lambda}}\right)-\hat{v}(0)\right), \quad \lambda \in l_{\varphi},
$$

где функция а определена выражением (3.13). Отметим, что в силу п. (5) утверждения 2.1 данные рассеяния $a(-\operatorname{sgn} E / \bar{\lambda})$ определены при $\lambda \in l_{\varphi}$. Используя п. (3) утверждения 2.1, получаем, что

$$
\frac{\partial \ln \Delta}{\partial \lambda}=\overline{u(\bar{\lambda})}, \quad \frac{\partial \ln \Delta}{\partial \bar{\lambda}}=u(\bar{\lambda}), \quad \lambda \in l_{\varphi}
$$

и

$$
\frac{\partial \ln \Delta}{\partial \gamma}=(u(\bar{\lambda})+\overline{u(\bar{\lambda})}) \cos \varphi+i(u(\bar{\lambda})-\overline{u(\bar{\lambda})}) \sin \varphi, \quad \gamma \in S^{1}, \gamma=(\cos \varphi, \sin \varphi)
$$

где $\frac{\partial \ln \Delta}{\partial \gamma}$ обозначает производную функции $\ln \Delta$ по направлению $\gamma$. Из (3.13) следует, что $u$ ограничена при $\lambda \rightarrow 0$. Таким образом, интегрируя (3.18) вдоль $l_{\varphi}$, мы получаем, что

$$
\ln \Delta(\lambda)=U(\bar{\lambda})+\overline{U(\bar{\lambda})}, \quad U(\bar{\lambda})=\int_{0}^{\bar{\lambda}} u(\bar{\zeta}) d \bar{\zeta}, \quad \lambda \in l_{\varphi},
$$

или

$$
\Delta(\lambda)=\Phi(\bar{\lambda}) \overline{\Phi(\bar{\lambda})}, \quad \text { где } \Phi(\bar{\lambda})=\exp U(\bar{\lambda}), \lambda \in l_{\varphi} .
$$


Из того, что $a$, заданная выражением $(3.13)$, определена на $\bar{l}_{\varphi}$, и из непрерывности $\Delta$ (см. п. (1) утверждения 2.1) следует, что представление (3.20) выполнено для $\lambda \in \bar{l}_{\varphi}$. В частности, из выражения (3.20) следует, что

$$
\Delta(\lambda) \neq 0 \text { для любого } \lambda \in \bar{l}_{\varphi} .
$$

Однако в силу определения (3.16) $\Delta\left(r^{\prime} e^{i \varphi}\right)=0$ для $r^{\prime} e^{i \varphi} \in \bar{l}_{\varphi}$, что противоречит (3.21). Таким образом, показано, что случай (ii) не может иметь места.

Итак, было доказано, что $\hat{v}(0)=0$ и, следовательно, $a(\lambda, 0) \equiv 0$ на $\lambda \in$ $\mathbb{C} \backslash(\mathscr{E} \cup 0)$.

Из уравнения $(2.18)$ и того факта, что $a \equiv 0$ на $\mathbb{C} \backslash \mathscr{E}$, вытекает, что $\Delta$ является голоморфной функцией на $\mathbb{C} \backslash(\mathscr{E} \cup T \cup 0)$, где $T=\{\lambda \in \mathbb{C}:|\lambda|=1\}$. Из пп. (1), (2) утверждения 2.1 следует, что $\Delta$ является голоморфной функцией на $\mathbb{C} \backslash(\mathscr{E} \cup T)$.

Предположим теперь, что $\mathscr{E} \neq \varnothing$. Поскольку $\mathscr{E}$ является замкнутым множеством, то существует $\lambda_{*} \in \mathscr{E}$, такое, что $\left|\lambda_{*}\right|=\min _{\lambda \in \mathscr{E}}|\lambda|$. Отметим, что, согласно п. (2) утверждения 2.1, $\left|\lambda_{*}\right|>0$.

Если $\left|\lambda_{*}\right| \geqslant 1$, то $\Delta(\lambda)$ является голоморфной функцией на $D_{+}=\{\lambda \in \mathbb{C}$ : $|\lambda|<1\}$, и из пп. (2), (3) утверждения 2.1 следует, что $\Delta \equiv 1$ на $D_{+}$. Если $\left|\lambda_{*}\right|<1$, то $\Delta(\lambda)$ - голоморфная функция на множестве $D_{+}^{h}=\{\lambda \in \mathbb{C}:$ $\left.|\lambda|<\lambda_{*}\right\}$, и из пп. (2), (3) следует, что $\Delta \equiv 1$ на $D_{+}^{h}$. С другой стороны, $\Delta\left(\lambda_{*}\right)=0$, что противоречит п. (1) утверждения 2.1. Таким образом, мы доказали, что $\Delta(\lambda) \equiv 1$ на $D_{+}$. Из п. (5) утверждения 2.1 вытекает, что $\Delta(\lambda) \equiv 1$ на $D_{-}=\{\lambda \in \mathbb{C}:|\lambda|>1\}$. Наконец, из п. (1) следует, что $\Delta \equiv 1$ на $\mathbb{C}$.

Функция $\mu$ является голоморфной на $\mathbb{C}$ в силу $(2.22),(2.23)$ и установленного факта, что $\mathscr{E}=\varnothing, b \equiv 0$. Функция $\mu$ также является ограниченной вследствие свойства (2.23). Из теоремы Лиувилля следует, что $\mu \equiv 1$. Наконец, из (2.24), (2.25) получаем, что $v \equiv 0$.

\section{§4. Доказательства лемм $3.2,3.3$}

Доказательство леммы 3.2. (1) Для доказательства п. (1) леммы 3.2 необходимо представить правую часть формулы (2.13) в виде суммы сходящегося ряда; тогда можно показать, что каждый член этого ряда для $\Delta_{\eta}(\lambda)$ совпадает с соответствующим членом ряда для $\Delta(\lambda)$.

(2) Формулы (3.5), (3.6) были выведены в [20] для случая отрицательной энергии. Здесь мы приводим их вывод одновременно для случая положительной и отрицательной энергии.

Отметим, что $\psi(z-\eta, \lambda)$ удовлетворяет $(2.1)$ с $v_{\eta}(z)$ и ведет себя асимптотически как

$$
\psi(z-\eta, \lambda)=e^{\frac{i \sqrt{E}}{2}(\lambda(\bar{z}-\bar{\eta})+(z-\eta) / \lambda)}(1+o(1))
$$

при $|z| \rightarrow \infty$. Таким образом, для собственных функций Фаддеева $\psi_{\eta}(z, \lambda)$, отвечающих потенциалу $v_{\eta}(z)$, получаем следующее представление: $\psi_{\eta}(z, \lambda)=$ $e^{\frac{i \sqrt{E}}{2}(\lambda \bar{\eta}+\eta / \lambda)} \psi(z-\eta, \lambda)$. Следовательно, для функции $\mu_{\eta}(z, \lambda)$, которая соответствует функции $\psi_{\eta}(z, \lambda)$ и определяется с помощью $(2.6)$, имеем $\mu_{\eta}(z, \lambda)=$ $\mu(z-\eta, \lambda)$.

Соответственно для данных рассеяния получаем

$a_{\eta}(\lambda)=\iint_{\mathbb{C}} v_{\eta}(\zeta) \mu_{\eta}(\zeta, \lambda) d \operatorname{Re} \zeta d \operatorname{Im} \zeta=\iint_{\mathbb{C}} v(\zeta-\eta) \mu(\zeta-\eta, \lambda) d \operatorname{Re} \zeta d \operatorname{Im} \zeta=a(\lambda)$ 
и

$$
\begin{aligned}
b_{\eta}(\lambda) & =\iint_{\mathbb{C}} \exp \left\{\frac{i \sqrt{E}}{2}\left(1+\frac{\operatorname{sgn} E}{\lambda \bar{\lambda}}\right)((\operatorname{sgn} E) \bar{\lambda} \zeta+\lambda \bar{\zeta})\right\} v_{\eta}(\zeta) \mu_{\eta}(\zeta, \lambda) d \operatorname{Re} \zeta d \operatorname{Im} \zeta \\
& =\iint_{\mathbb{C}} \exp \left\{\frac{i \sqrt{E}}{2}\left(1+\frac{\operatorname{sgn} E}{\lambda \bar{\lambda}}\right)((\operatorname{sgn} E) \bar{\lambda} \zeta+\lambda \bar{\zeta})\right\} v(\zeta-\eta) \mu(\zeta-\eta, \lambda) d \operatorname{Re} \zeta d \operatorname{Im} \zeta \\
& =\exp \left\{\frac{i \sqrt{E}}{2}\left(1+\frac{\operatorname{sgn} E}{\lambda \bar{\lambda}}\right)((\operatorname{sgn} E) \bar{\lambda} \eta+\lambda \bar{\eta})\right\} b(\lambda) .
\end{aligned}
$$

Аналогично, чтобы получить формулы $(3.7),(3.8)$, отметим, что $\varphi(z-\eta, \lambda)$ удовлетворяет $(2.1)$ с $v_{\eta}(z)$ и ведет себя асимптотически как

$$
\varphi(z-\eta, \lambda)=e^{\frac{i \sqrt{E}}{2}(\lambda(\bar{z}-\bar{\eta})+(z-\eta) / \lambda)}\left(\frac{i \sqrt{E}}{2}\left(\lambda(\bar{z}-\bar{\eta})-\frac{1}{\lambda}(z-\eta)\right)+o(1)\right)
$$

при $|z| \rightarrow \infty$. Таким образом, для собственной функции $\varphi_{\eta}(z, \lambda)$ уравнения $(2.1)$ с потенциалом $v_{\eta}(z)$, удовлетворяющей асимптотике $(2.7)$, получаем следующее представление: $\varphi_{\eta}(z, \lambda)=e^{\frac{i \sqrt{E}}{2}(\lambda \bar{\eta}+\eta / \lambda)}\left(\varphi(z-\eta, \lambda)+\frac{i \sqrt{E}}{2}\left(\lambda \bar{\eta}-\frac{1}{\lambda} \eta\right) \psi(z-\eta, \lambda)\right)$. Следовательно, для функции $\nu_{\eta}(z, \lambda)$, которая соответствует функции $\varphi_{\eta}(z, \lambda)$ и определяется в $(2.7)$, имеем $\nu_{\eta}(z, \lambda)=\nu(z-\eta, \lambda)+\frac{i \sqrt{E}}{2}\left(\lambda \bar{\eta}-\frac{1}{\lambda} \eta\right) \mu(z-\eta, \lambda)$.

Соответственно для данных рассеяния получаем

$$
\begin{gathered}
\alpha_{\eta}(\lambda)=\iint_{\mathbb{C}} v_{\eta}(\zeta) \nu_{\eta}(\zeta, \lambda) d \operatorname{Re} \zeta d \operatorname{Im} \zeta=\iint_{\mathbb{C}} v(\zeta-\eta) \nu(\zeta-\eta, \lambda) d \operatorname{Re} \zeta d \operatorname{Im} \zeta \\
+\frac{i \sqrt{E}}{2}\left(\lambda \bar{\eta}-\frac{\eta}{\lambda}\right) \iint_{\mathbb{C}} v(\zeta-\eta) \mu(\zeta-\eta, \lambda) d \operatorname{Re} \zeta d \operatorname{Im} \zeta=\alpha(\lambda)+\frac{i \sqrt{E}}{2}\left(\lambda \bar{\eta}-\frac{\eta}{\lambda}\right) a(\lambda)
\end{gathered}
$$

и

$$
\begin{gathered}
\beta_{\eta}(\lambda)=\iint_{\mathbb{C}} \exp \left\{\frac{i \sqrt{E}}{2}\left(1+\frac{\operatorname{sgn} E}{\lambda \bar{\lambda}}\right)((\operatorname{sgn} E) \bar{\lambda} \zeta+\lambda \bar{\zeta})\right\} v_{\eta}(\zeta) \nu_{\eta}(\zeta, \lambda) d \operatorname{Re} \zeta d \operatorname{Im} \zeta \\
=\iint_{\mathbb{C}} \exp \left\{\frac{i \sqrt{E}}{2}\left(1+\frac{\operatorname{sgn} E}{\lambda \bar{\lambda}}\right)((\operatorname{sgn} E) \bar{\lambda} \zeta+\lambda \bar{\zeta})\right\} v(\zeta-\eta) \nu(\zeta-\eta, \lambda) d \operatorname{Re} \zeta d \operatorname{Im} \zeta \\
+\frac{i \sqrt{E}}{2}\left(\lambda \bar{\eta}-\frac{\eta}{\lambda}\right) \iint_{\mathbb{C}} \exp \left\{\frac{i \sqrt{E}}{2}\left(1+\frac{\operatorname{sgn} E}{\lambda \bar{\lambda}}\right)((\operatorname{sgn} E) \bar{\lambda} \zeta+\lambda \bar{\zeta})\right\} \\
\times v(\zeta-\eta) \mu(\zeta-\eta, \lambda) d \operatorname{Re} \zeta d \operatorname{Im} \zeta \\
=\exp \left\{\frac{i \sqrt{E}}{2}\left(1+\frac{\operatorname{sgn} E}{\lambda \bar{\lambda}}\right)((\operatorname{sgn} E) \bar{\lambda} \eta+\lambda \bar{\eta})\right\}\left(\beta(\lambda)+\frac{i \sqrt{E}}{2}\left(\lambda \bar{\eta}-\frac{\eta}{\lambda}\right) b(\lambda)\right) .
\end{gathered}
$$

Для того чтобы доказать лемму 3.3 , рассмотрим оператор

$$
T=\partial_{t}-8 \partial_{z}^{3}-2 w \partial_{z}-8 \partial_{\bar{z}}^{3}-2 \bar{w} \partial_{\bar{z}},
$$

где функция $w$ определена в $(1.1 \mathrm{~b}),(1.8)$ для некоторого потенциала $v$. Отметим, что $T=\partial_{t}+A$, где $A-$ оператор из (1.5).

Нам понадобится следующая вспомогательная лемма, описывающая, как $T$ действует на спектральные решения двумерного уравнения Шрёдингера. 
Лемма 4.1. Пусть $(v, w)$ удовлетворяет условиям (1.6)-(1.8) и

$$
\left|\partial_{t} v(x, t)\right| \leqslant \frac{\tilde{q}(t)}{(1+|x|)^{3+\varepsilon}} \quad \text { для некоторого } \tilde{q}(t)>0 \text {. }
$$

Предположим, что для некоторого $\lambda \in \mathbb{C},|\lambda| \neq 1$, и для $t$, принадлежащих определенному интервалу $t \in\left(t_{1}, t_{2}\right)$, решение $\psi(z, \lambda, t)$ уравнения $(1.3) c$ асимптотикой (2.6) существует и единственно. Аналогично, предположим, что решение $\varphi(z, \lambda, t)$ уравнения (1.3) с асимптотикой (2.7) существует $u$ единственно. Тогда

$$
\begin{aligned}
& T \psi=i(\sqrt{E})^{3} e^{\frac{i \sqrt{E}}{2}(\lambda \bar{z}+z / \lambda)}\left(\left(\lambda^{3}+\frac{1}{\lambda^{3}}\right)+o(1)\right) \quad \text { npu }|z| \rightarrow \infty \\
& T \varphi=i(\sqrt{E})^{3} e^{\frac{i \sqrt{E}}{2}}(\lambda \bar{z}+z / \lambda)\left(\frac{i \sqrt{E}}{2}\left(\lambda^{3}+\frac{1}{\lambda^{3}}\right)\left(\lambda \bar{z}-\frac{z}{\lambda}\right)+3\left(\lambda^{3}-\frac{1}{\lambda^{3}}\right)+o(1)\right) \\
& \text { npu }|z| \rightarrow \infty .
\end{aligned}
$$

Доказательство. Прежде всего, вследствие леммы 3.1 для доказательства соотношений $(4.3),(4.4)$ достаточно показать, что

$$
\begin{array}{r}
\partial_{t} \mu \rightarrow 0, \partial_{z}^{j} \mu \rightarrow 0, \partial_{\bar{z}}^{j} \mu \rightarrow 0, \quad j=1,2,3, \quad \text { при }|z| \rightarrow \infty, \\
\partial_{t} \nu \rightarrow 0, \partial_{z} \nu \rightarrow-\frac{i \sqrt{E}}{2 \lambda}, \partial_{\bar{z}} \nu \rightarrow \frac{i \sqrt{E}}{2} \lambda, \partial_{z}^{k} \nu \rightarrow 0, \partial_{\bar{z}}^{k} \nu \rightarrow 0, \quad k=2,3, \\
\text { при }|z| \rightarrow \infty,
\end{array}
$$

где $\mu(z, \lambda, t)=e^{-\frac{i \sqrt{E}}{2}(\lambda \bar{z}+z / \lambda)} \psi(z, \lambda, t), \nu(z, \lambda, t)=e^{-\frac{i \sqrt{E}}{2}(\lambda \bar{z}+z / \lambda)} \varphi(z, \lambda, t)$. Мы докажем только свойства (4.5). Свойства (4.6) доказываются аналогично.

Функция $\mu$ определяется как решение интегрального уравнения (2.8), где используется обозначение (2.10). Дифференцируя (2.8) по $t$, получаем следующее интегральное уравнение для $\partial_{t} \mu$ :

$$
\begin{aligned}
\partial_{t} \mu(z, \lambda, t)=\iint_{\mathbb{C}} g(z-\zeta, \lambda) \partial_{t} v(\zeta, t) \mu(\zeta, \lambda, t) d \operatorname{Re} \zeta d \operatorname{Im} \zeta \\
+\iint_{\mathbb{C}} g(z-\zeta, \lambda) v(\zeta, t) \partial_{t} \mu(\zeta, \lambda, t) d \operatorname{Re} \zeta d \operatorname{Im} \zeta
\end{aligned}
$$

Дифференцируя (2.8) j раз по z, получаем следующее интегральное уравнение для $\partial_{z}^{j} \mu$ :

$$
\partial_{z}^{j} \mu(z, \lambda, t)=\iint_{\mathbb{C}} \partial_{z}^{j} g(z-\zeta, \lambda) v(\zeta, t) \mu(\zeta, \lambda, t) d \operatorname{Re} \zeta d \operatorname{Im} \zeta, \quad j=1,2,3 .
$$

Проинтегрируем правую часть по частям, учитывая свойство (1.7) функции $v$ и то, что $\partial_{z}^{j} g(z-\zeta, \lambda)=(-1)^{j} \partial_{\zeta}^{j} g(z-\zeta, \lambda)$. Таким образом, получаем

$$
\partial_{z}^{j} \mu(z, \lambda, t)=\iint_{\mathbb{C}} g(z-\zeta, \lambda) \partial_{\zeta}^{j}(v(\zeta, t) \mu(\zeta, \lambda, t)) d \operatorname{Re} \zeta d \operatorname{Im} \zeta, \quad j=1,2,3 .
$$

Аналогично показывается, что $\partial_{\bar{z}}^{j} \mu$ удовлетворяет интегральному уравнению

$$
\partial_{\bar{z}}^{j} \mu(z, \lambda, t)=\iint_{\mathbb{C}} g(z-\zeta, \lambda) \partial_{\bar{\zeta}}^{j}(v(\zeta, t) \mu(\zeta, \lambda, t)) d \operatorname{Re} \zeta d \operatorname{Im} \zeta, \quad j=1,2,3
$$


Уравнение (4.8) является уравнением на неизвестную функцию $\partial_{z}^{j} \mu$ : предполагается, что функции $\partial_{z}^{k} \mu, k<j$, определены ранее. Аналогично, уравнение (4.9) является уравнением на неизвестную функцию $\partial_{z}^{j} \mu$, где предполагается, что функции $\partial_{\bar{z}}^{k} \mu, k<j$, определены ранее. Из предположений леммы следует, что для каждого из уравнений (4.7)-(4.9) решение существует, единственно и может быть представлено в виде $(1+|z|)^{2+\varepsilon / 2} u(z, \lambda, t)$ для некоторой подходящей функции $u(\cdot, \lambda, t) \in L^{2}(\mathbb{C})$.

В [23] было показано, что функция $g$, определенная в (2.10), обладает следующим свойством: $|g(z, \lambda)| \leqslant$ const $/|z|$ при любом $\lambda \in \mathbb{C},|\lambda| \neq 1$, и достаточно больших $z$, и $|g(z, \lambda)| \leqslant$ const $|\ln | z||$ при любом $\lambda \in \mathbb{C},|\lambda| \neq 1$, и достаточно малых $z$. Из этого свойства следует, что

$$
\left|\iint_{\mathbb{C}} g(z-\zeta, \lambda) U(\zeta) d \operatorname{Re} \zeta d \operatorname{Im} \zeta\right| \rightarrow 0 \quad \text { при }|z| \rightarrow \infty
$$

для любой функции $U \in L^{1}(\mathbb{C}) \cap L^{2}(\mathbb{C})$.

Обозначим через $\xi(z, \lambda, t)$ решение любого из уравнений $(2.8),(4.7)-(4.9)$. Как указано выше, $\xi(z, \lambda, t)$ может быть представлено в виде $\xi(z, \lambda, t)=$ $(1+|z|)^{2+\varepsilon / 2} u(z, \lambda, t)$ для некоторой функции $u(\cdot, \lambda, t) \in L^{2}(\mathbb{C})$. Тогда из предположений на функцию $v$ следует, что $\partial_{z}^{j} v(\cdot, t) \xi(\cdot, \lambda, t) \in L^{1}(\mathbb{C}) \cap L^{2}(\mathbb{C})$ и $\partial_{\bar{z}}^{j} v(\cdot, t) \xi(\cdot, \lambda, t) \in L^{1}(\mathbb{C}) \cap L^{2}(\mathbb{C})$ при $j=0, \ldots, 3$ и $\partial_{t} v(\cdot, t) \xi(\cdot, \lambda, t) \in L^{1}(\mathbb{C}) \cap L^{2}(\mathbb{C})$. Таким образом, из $(4.10)$ при $U(\cdot)=\partial_{t} v(\cdot, t) \mu(\cdot, \lambda, t)$ и $U(\cdot)=v(\cdot, t) \partial_{t} \mu(\cdot, \lambda, t)$ следует, что правая часть в (4.7) стремится к нулю при $|z| \rightarrow \infty$. Аналогично, рассматривая уравнения (4.8), (4.9) последовательно, мы получаем, что правая часть каждого из них стремится к нулю при $|z| \rightarrow \infty$. Следовательно, $\partial_{t} \mu \rightarrow 0$, $\partial_{z}^{j} \mu \rightarrow 0, \partial_{\bar{z}}^{j} \mu \rightarrow 0, j=1,2,3$, при $|z| \rightarrow \infty$.

Доказательство леммы 3.3. Формулы (3.9), (3.10) известны (см., например, [12]). Поскольку их вывод аналогичен выводу формул (3.11), (3.12), мы ограничимся выводом последних. Вывод аналогов формул (3.9)-(3.12) для случая нулевой энергии можно найти в [2].

Уравнение (1.1) представляет условие, при котором

$$
[T, L] \eta=E T \eta \quad \text { для любой функции } \eta \text {, такой, что } L \eta=E \eta \text {, }
$$

где оператор $L$ определен в (2.1), а оператор $T$ - в (4.2) (см. [22], [2]).

Положим $\eta=\varphi$, где $\varphi$ - решение уравнения $L \varphi=E \varphi$ с асимптотикой (2.7). Тогда из (4.11) следует, что $L T \varphi=E \varphi$. Из леммы 4.1 получаем, что

$$
\begin{array}{r}
T \varphi=i(\sqrt{E})^{3} e^{\frac{i \sqrt{E}}{2}(\lambda \bar{z}+z / \lambda)}\left(\frac{i \sqrt{E}}{2}\left(\lambda^{3}+\frac{1}{\lambda^{3}}\right)\left(\lambda \bar{z}-\frac{z}{\lambda}\right)+3\left(\lambda^{3}-\frac{1}{\lambda^{3}}\right)+o(1)\right), \\
|z| \rightarrow \infty
\end{array}
$$

Из единственности решения уравнения (2.1) с асимптотикой (2.6) при рассматриваемом значении $\lambda$ вытекает, что

$$
T \varphi=i(\sqrt{E})^{3}\left(\lambda^{3}+\frac{1}{\lambda^{3}}\right) \varphi+3 i(\sqrt{E})^{3}\left(\lambda^{3}-\frac{1}{\lambda^{3}}\right) \psi .
$$


Другими словами,

$\partial_{t} \varphi=8 \partial_{z}^{3} \varphi+2 w \partial_{z} \varphi+8 \partial_{\bar{z}}^{3} \varphi+2 \bar{w} \partial_{\bar{z}} \varphi+i(\sqrt{E})^{3}\left(\lambda^{3}+\frac{1}{\lambda^{3}}\right) \varphi+3 i(\sqrt{E})^{3}\left(\lambda^{3}-\frac{1}{\lambda^{3}}\right) \psi$.

Теперь запишем функцию $\alpha(\lambda, t)$ в виде

$$
\alpha(\lambda, t)=\iint_{\mathbb{C}} e^{-\frac{i \sqrt{E}}{2}(\lambda \bar{\zeta}+\zeta / \lambda)} v(\zeta, t) \varphi(\zeta, \lambda, t) d \operatorname{Re} \zeta d \operatorname{Im} \zeta
$$

и вычислим ее производную по времени:

$$
\begin{aligned}
& \partial_{t} \alpha(\lambda, t)=\iint_{\mathbb{C}} e^{-\frac{i \sqrt{E}}{2}(\lambda \bar{\zeta}+\zeta / \lambda)} \partial_{t} v(\zeta, t) \varphi(\zeta, \lambda, t) d \operatorname{Re} \zeta d \operatorname{Im} \zeta \\
&+\iint_{\mathbb{C}} e^{-\frac{i \sqrt{E}}{2}(\lambda \bar{\zeta}+\zeta / \lambda)} v(\zeta, t) \partial_{t} \varphi(\zeta, \lambda, t) d \operatorname{Re} \zeta d \operatorname{Im} \zeta .
\end{aligned}
$$

Подставляя (1.1) и (4.12) в (4.13), интегрируя получающееся уравнение по частям и учитывая, что $-4 \partial_{\zeta} \partial_{\bar{\zeta}} \varphi+v \varphi=E \varphi$, получаем

$$
\partial_{t} \alpha(\lambda, t)=3 i(\sqrt{E})^{3}\left(\lambda^{3}-\frac{1}{\lambda^{3}}\right)(a(\lambda, t)-\hat{v}(0))
$$

(подробные вычисления для вывода уравнения (4.14) см. в электронной версии данной статьи [18]). Из формул (3.9), (4.14) вытекает (3.11).

Аналогично, запишем функцию $\beta(\lambda, t)$ в виде

$$
\beta(\lambda, t)=\iint_{\mathbb{C}} e^{\frac{\sqrt{-E}}{2}(\bar{\lambda} \zeta+\bar{\zeta} / \bar{\lambda})} v(\zeta, t) \varphi(\zeta, \lambda, t) d \operatorname{Re} \zeta d \operatorname{Im} \zeta
$$

и вычислим ее производную по времени:

$$
\begin{aligned}
& \partial_{t} \beta(\lambda, t)=\iint_{\mathbb{C}} e^{\frac{\sqrt{-E}}{2}(\bar{\lambda} \zeta+\bar{\zeta} / \bar{\lambda})} \partial_{t} v(\zeta, t) \varphi(\zeta, \lambda, t) d \operatorname{Re} \zeta d \operatorname{Im} \zeta \\
&+\iint_{\mathbb{C}} e^{\frac{\sqrt{-E}}{2}(\bar{\lambda} \zeta+\bar{\zeta} / \bar{\lambda})} v(\zeta, t) \partial_{t} \varphi(\zeta, \lambda, t) d \operatorname{Re} \zeta d \operatorname{Im} \zeta .
\end{aligned}
$$

Подставляя (1.1) и (4.12) в (4.15), интегрируя получающееся выражение по частям и учитывая, что $-4 \partial_{\zeta} \partial_{\bar{\zeta}} \varphi+v \varphi=E \varphi$, получаем

$$
\begin{aligned}
\partial_{t} \beta(\lambda, t)=i(\sqrt{E})^{3}\left(\lambda^{3}+\frac{1}{\lambda^{3}}+(\operatorname{sgn} E)\left(\bar{\lambda}^{3}\right.\right. & \left.\left.+\frac{1}{\bar{\lambda}^{3}}\right)\right) \beta(\lambda, t) \\
& +3 i(\sqrt{E})^{3}\left(\lambda^{3}-\frac{1}{\lambda^{3}}\right) b(\lambda, t)
\end{aligned}
$$

(подробные вычисления для вывода уравнения (4.16) можно найти в электронной версии данной статьи [18]). Используя формулу (3.10), получаем (3.12).

\section{ЛитерАТУРА}

[1] M. J. Ablowitz, P. A. Clarkson, Solitons, nonlinear evolution equations and inverse scattering, Cambridge University Press, Cabridge, 1991.

[2] M. Boiti, J.J.-P. Leon, M. Manna, F. Pempinelli, On a spectral transform of a $K d V$ like equation related to the Schrödinger operator in the plane, Inverse Problems, 3:1 (1987), 25-36. 
[3] M. Boiti, J.J.-P. Leon, L. Martina, F. Pempinelli, Scattering of localized solitons in the plane, Phys. Lett. A., 132 (1988), 432-439.

[4] A. de Bouard, J.-C. Saut, Solitary waves of generalized Kadomtsev-Petviashvili equations, Ann. Inst. H. Poincaré, Analyse Non Linéaire, 14:2 (1997), 211-236.

[5] A. de Bouard, J.-C. Saut, Symmetries and decay of the generalized KadomtsevPetviashvili solitary waves, SIAM J. Math. Anal., 28:5 (1997), 1064-1085.

[6] Л. Д. Фаддеев, Растущие решения уравнения Шрёдингера, Докл. АН СССР, 165 (1965), 514-517.

[7] Л. Д. Фаддеев, Обратная задача квантовой теории рассеяния. II, в кн.: Итоги науки и техники. Современные проблемы математики, т. 3, ВИНИТИ, М., 1974, 93-180.

[8] A. S. Fokas, M. J. Ablowitz, On the inverse scattering of the time-dependent Schrödinger equation and the associated Kadomtsev-Petviashvili equation, Stud. Appl. Math., 69:3 (1983), 211-228.

[9] A. S. Fokas, P. M. Santini, Coherent structures in multidimensions, Phys. Rev. Lett., 63:13 (1983), 1329-1333.

[10] И. Ц. Гохберг, М. Г. Крейн, Введение в теорию линейных несамосопряженных операторов в гилъбертовом пространстве, Наука, М., 1965.

[11] П. Г. Гриневич, Рациональные солитоны уравнений Веселова-Новикова - безотражательные при фиксированной энергии двумерные потенииаль, ТМФ., 69:2 (1986), 307-310.

[12] П. Г. Гриневич, Преобразование рассеяния для двумерного оператора Шрёдингера с убывающим на бесконечности потенциалом при фиксированной ненулевой энергии, УМН, 55:6(336) (2000), 3-70.

[13] П. Г. Гриневич, Р. Г. Новиков, Аналоги многосолитонных потенциалов для двумерного оператора Шрёдингера и нелокальная задача Римана, Докл. АН СССР, 286:1 (1986), 19-22.

[14] П. Г. Гриневич, С. П. Новиков, Двумерная «обратная задача рассеяния» для отрицательных энергий и обобщенно-аналитические функиии. I. Энергии ниже основного состояния, Функц. анализ и его прил., 22:1 (1988), 23-33.

[15] Р. Г. Новиков, Г. М. Хенкин, д̄-уравнение в многомерной обратной задаче рассеяния, УМH, 42:3 (1987), 93-152.

[16] A. V. Kazeykina, A large time asymptotics for the solution of the Cauchy problem for the Novikov-Veselov equation at negative energy with non-singular scattering data, Inverse Problems, 28:5 (2012), 055017.

[17] А. В. Казейкина, Отсутствие солитонов кондуктивного типа для уравнения Веселова-Новикова при нулевой энергии, Функц. анализ и его прил., 47:1 (2013), 79-82.

[18] A. V. Kazeykina, Absence of solitons with sufficient algebraic localization for the Novikov-Veselov equation at nonzero energy, http://arxiv.org/abs/1201.2758.

[19] A. V. Kazeykina, R. G. Novikov, A large time asymptotics for transparent potentials for the Novikov-Veselov equation at positive energy, J. Nonlinear Math. Phys., 18:3 (2011), 377-400.

[20] A. V. Kazeykina, R. G. Novikov, Large time asymptotics for the Grinevich-Zakharov potentials, Bull. Sci. Math., 135:4 (2011), 374-382.

[21] A. V. Kazeykina, R. G. Novikov, Absence of exponentially localized solitons for the Novikov-Veselov equation at negative energy, Nonlinearity, 24:6 (2011), 1821-1830.

[22] С. В. Манаков, Метод обратной задачи рассеяния и двумерные эволючионные уравнения, УМН, 31:5 (1976), 245-246.

[23] R. G. Novikov, The inverse scattering problem on a fixed energy level for the twodimensional Schrödinger operator, J. Funct. Anal., 103:2 (1992), 409-463. 
[24] R. G. Novikov, Absence of exponentially localized solitons for the Novikov-Veselov equation at positive energy, Physics Letters A, 375:9 (2011), 1233-1235.

[25] А. П. Веселов, С. П. Новиков, Конечнозонные двумерные потенииалъные операторы Шрёдингера. Явные формуль и эволюиионные уравнения, Докл. АН СССР, 279:1 (1984), 20-24.

[26] А. П. Веселов, С. П. Новиков, Конечнозонные двумерные потенииалъные операторы Шрёдингера. Потенциалъные операторы, Докл. АН СССР, 279:4 (1984), 784-788.

Московский государственный университет

CMAP, Ecole Polytechnique, France

Поступило в редакцию

e-mail: kazeykina@cmap.polytechnique.fr 20 января 2012 г. 